University of Nebraska - Lincoln

DigitalCommons@University of Nebraska - Lincoln

Ralph Skomski Publications

Research Papers in Physics and Astronomy

September 1996

\title{
An Effective-Viscosity Description of Domain-Wall Motion in Magnetic Thin Films with Perpendicular Anisotropy
}

\author{
Ralph Skomski \\ University of Nebraska-Lincoln, rskomski2@unl.edu \\ J. Giergiel \\ Max-Planck-Institut fur Mikrostrukturphysik, Weinberg 2, 06120 Halle, Germany \\ J. Kirschner \\ Max-Planck-Institut fur Mikrostrukturphysik, Weinberg 2, 06120 Halle, Germany
}

Follow this and additional works at: https://digitalcommons.unl.edu/physicsskomski

Part of the Physics Commons

Skomski, Ralph; Giergiel, J.; and Kirschner, J., "An Effective-Viscosity Description of Domain-Wall Motion in Magnetic Thin Films with Perpendicular Anisotropy" (1996). Ralph Skomski Publications. 8.

https://digitalcommons.unl.edu/physicsskomski/8

This Article is brought to you for free and open access by the Research Papers in Physics and Astronomy at DigitalCommons@University of Nebraska - Lincoln. It has been accepted for inclusion in Ralph Skomski Publications by an authorized administrator of DigitalCommons@University of Nebraska - Lincoln. 


\title{
An Effective-Viscosity Description of Domain-Wall Motion in Magnetic Thin Films with Perpendicular Anisotropy
}

\author{
R. Skomski, J. Giergiel, and J. Kirschner \\ Max-Planck-Institut für Mikrostrukturphysik, Weinberg 2, 06120 Halle, Germany
}

\begin{abstract}
The expansion of magnetic domains in thin films with perpendicular anisotropy is investigated. To determine the domain-wallvelocity as a function of the applied magnetic field a selfconsistent magnetic-viscosity approach is used. The main predictions of the theory are a linear behaviour in the limit of moderately strong fields, a quasi-exponential behaviour for fields close to the propagation field, and a negative velocity for very small and negative fields. The predictions of the theory are compatiblle with domain-wall investigations on $\mathrm{Au} / \mathrm{Co} / \mathrm{Au}$ sandwiches.
\end{abstract}

\section{INTRODUCTION}

The time dependence of the magnetization of ultrathin films with perpendicular anisotropy is a scientifically interesting and - in the context of magneto-optical recording - technologically relevant subject. In [1], magnetization measurements have been used to investigate the magnetic aftereffect in hcp cobalt sandwiched between $\mathrm{Au}(111)$ thin films. A more recent series of papers [2-5] dealing with $\mathrm{Au} / \mathrm{Co} / \mathrm{Au}$ thin films is devoted to direct domain observations by Faraday rotation. Another example are 3 to 4 monolayer films of $\gamma-\mathrm{Fe}$ on $\mathrm{Cu}(001)$, where magneto-optical Kerr microscopy has been used to make magnetic aftereffect and domain observations $[6,7]$.

Compared to the magnetic aftereffect in bulk materials, where it is very difficult to monitor reversed domains, Kerr and Faraday micrographs of ultrathin films give a direct insight into the domain structure. As a consequence, it is not necessary to restrict the attention to the time dependence of the magnetization, which is of ten fitted to a logarihmic law $\Delta \mathrm{M} \propto \ln \mathrm{t}[8-11]$. Figure 1 gives a schematic idea of the domain structure in a low-purity and $\mathbf{b}$ high-purity films. In high-purity films, the density of reversed domains is low, and it is possible to measure the size of individual domains as a function function of time and magnetic field $[3,5]$.

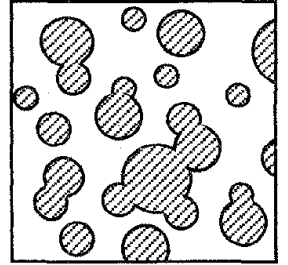

a

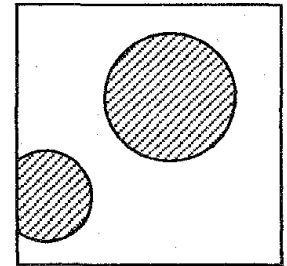

b

Fig. 1. Reversed domains (schematic): a high density of nuclei and $\mathbf{b}$ low density of nuclei.

Manuscript received March 3, 1996. 
Magnetic hysteresis is a non-equilibrium phenomenon that reflects the existence of at least two energy minima. The magnetic aftereffect, also called aging or magnetic viscosity, arises from thermally activated changes of the magnetization state. In spite of numerous attempts to describe magnetic viscosity and domain-wall motion, there is no comprehensive microscopic theory of these phenomena. Only in a few cases it is possible to derive exact magneticviscosity results. For example, aligned Stoner-Wohlfarth particles [13] are characterized by the trivial energy barrier

$$
\Delta \mathrm{E}=\mathrm{K}_{1} \mathrm{~V}\left(1-\frac{\mathrm{H}}{\mathrm{H}_{\mathrm{a}}}\right)^{2}
$$

so that the magnetization of fine particles approaches its equilibrium state with the relaxation time $\tau=\tau_{0}$ $\exp (\Delta \mathrm{E} / \mathrm{kBT})$. For domain-wall pinning at isolated defects (strong pinning) one obtains $\Delta \mathrm{E} \propto\left(1-\mathrm{H} / \mathrm{H}_{\mathrm{c}}\right)^{3 / 2}[10]$, and it can be shown that this exponent $3 / 2$ remains valid whenever the domain-wall energy can be expanded into powers of the wall position (see Appendix). However, to avoid the detailed discussion of the field dependence of the magnetic energy it is common to use quasi-phenomenological exponential laws of the type

$$
\mathrm{v}=\mathrm{v}^{\prime} \mathrm{e}^{\mathrm{H} / \mathrm{H}_{\mathrm{o}}}
$$

to fit the experimental data $[1,3,5,7]$. Note that Eq. (2) can be derived from more complicated equations by linearizing the magnetic energy' with respect to $H$.

\section{Calculation AND RESULTS}

Domain-wall expansion is not continuous but proceeds by discrete Barkhausen jumps. For instance, the Barkhausen volume VB predicted by the Stoner-Wohlfarth model equals the volume $V$ of the interaction-free particles. The aftereffect caused by domain-wall motion is much more difficult to analyze, since it involves energy barrier distributions and interaction effects [8-10]. However, if the Barkhausen length associated with the discontinuous domain-wall motion is much smaller than the domain size, then one can treat the film as a continuum. Typical domain sizes in ultrathin films with perpendicular anisotropy are of order $10 \mu \mathrm{m}$, as compared to Barkhausen lengths smaller than about $100 \mathrm{~nm}$ [1], so that the macroscopic approach is a reasonable approximation.

Consider a circular domain in a magnetic thin film of thickness $\mathrm{L}$. The starting point is the magnetic energy

$$
\frac{E}{L}=2 \pi R \gamma(R)-2 \pi R^{2} \mu_{0} M_{S} H
$$

Here $\mathrm{R}$ is the domain radius and $\gamma(\mathrm{R})$ denotes the domainwall energy averaged over the periphery of the circle: $\gamma(R)=$ $(1 / 2 \pi) \int \gamma(\mathrm{R}, \phi) \mathrm{d} \phi$. Of course, the replacement of real domains by circular domains means that the present approach is not suitable to investigate the fractality [3] of the wall. Replacing the detailed interaction of the domain-wall spins by a viscous medium of domain-wall viscosity $\eta_{0}$ yields

$$
\frac{\mathrm{dR}}{\mathrm{dt}}=-\frac{1}{2 \pi \mathrm{LR} \eta_{\mathrm{o}}} \frac{\partial \mathrm{E}}{\partial \mathrm{R}}
$$

Since the wall energy $\gamma(R)$ is a random function of $R$, it is convenicnt to introduce a locally averaged wall energy $\langle\gamma\rangle=$ $(1 / \Delta R) \int \gamma(R) d R$, where $\Delta R$ is much larger than the Barkhausen length. Separating $\langle\gamma\rangle$ from the remaining random contribution $\Delta \gamma$ associated with local inhomogenities yields $\gamma(\mathrm{R})=\langle\gamma\rangle+\Delta \gamma(\mathrm{R})$,

$$
\frac{1}{\mathrm{~L}} \frac{\partial \mathrm{E}}{\partial \mathrm{R}}=2 \pi \gamma_{\mathrm{o}}-4 \pi \mathrm{R} \mu_{\mathrm{o}} \mathrm{M}_{\mathrm{S}} \mathrm{H}+2 \pi \frac{\partial(\mathrm{R} \Delta \gamma)}{\partial \mathrm{R}}
$$

and

$$
\frac{\mathrm{dR}}{\mathrm{dt}}=\mu\left(\mathrm{H}-\mathrm{H}_{\mathrm{p}}\right)-\mathrm{f}(\mathrm{R})
$$

Here we have introduced reduced parameters: $\mu=2 \mu_{\mathrm{o}} \mathrm{M}_{\mathrm{s}} / \eta_{\mathrm{o}}$ is an average domain-wall mobility, $\mathrm{H}_{\mathrm{p}}=\gamma_{\mathrm{o}} / 2 \mathrm{R} \mu_{\mathrm{O}} \mathrm{M}_{\mathrm{S}}$ is the propagation field deduced from the average wall encrgy $\gamma_{O}$, and

$$
f(R)=\frac{1}{L R \eta_{O}} \frac{\partial(R \Delta y)}{\partial R}
$$

may be interpreted as a random force acting on the wall.

The randomness of $f(R)$, which arises from the random character of the domain-wall energy $\gamma(R)$, complicates the determination of the function $R(t)$. To specify the problem, it is comparatively easy to determine the reverse function $t(R)$ for a given configuration $f(R)$ of random forces, but due to the involvement of $f(R)$ it is nearly impossible to calculate the sought-for original function $R(t)$ from $t(R)$. However, the use of $t(R)$ has two advantages. First, since the length scale $\Delta R$ relevant to Kerr or Faraday microscopy is much larger than the scale of the structural inhomogenities and the Barkhausen length, it is sufficient to consider the average time of expansion $\langle l\rangle=(1 / \Delta R) \int t(R) d R$. Secondly, it is possible to expand $t(R)$ into powers of $f(R)$, which can then be replaced by averages $\left\langle\mathrm{f}^{\mathrm{m}}(R)>\right.$.

Up to second order, this expansion procedure leads to a renormalization of the domain-wall viscosity

$$
\eta(H)=\eta_{0}\left(1+\frac{q^{2}}{\mu^{2}(H-H p)^{2}}\right)
$$

Here the 'self-energy' parameter $q^{2}=\left\langle f^{2}(R)\right\rangle$ describes the micromagnetic inhomogenity of the film. Equation (8) shows that the reduction of the domain-wall mobility $\mu$ due to pinning is equivalent to an enhancement of the viscosity. The domain-wall velocity $v=\Delta R /\langle\Delta$ is

$$
v=\frac{\mu^{3}\left(\mathrm{H}-\mathrm{H}_{\mathrm{p}}\right)^{3}}{\left(\mathrm{q}^{2}+\mu^{2}\left(\mathrm{H}-\mathrm{H}_{\mathrm{p}}\right)^{2}\right)}
$$

From Eqs. (8) and (9) we see that $\eta=\infty$ and $v=0$ for $H=$ $\mathrm{H}_{\mathrm{p}}$ - pinning is complete if the external field equals the average propagation field.

\section{COMPARISON WITH EXPERIMENT}

Figure 3 compares the theoretical prediction Eq. (9) with measurements on $10 \AA$ hcp Co sandwiched between Au(111) thin films. The data points have been obtained from Faraday micrographs at $318 \mathrm{~K}$ [4, 5], whereas the solid line is a leastsquare fit of the experimental data. The fitting parameters are $\mu_{\mathrm{o}} \mathrm{H}_{\mathrm{p}}=69.5 \mathrm{mT}, \mu / \mu_{\mathrm{O}}=1.41 \mathrm{~m} /(\mathrm{s} . \mathrm{mT})$, and $\mu=19.1 \mathrm{~m} / \mathrm{s}$. 


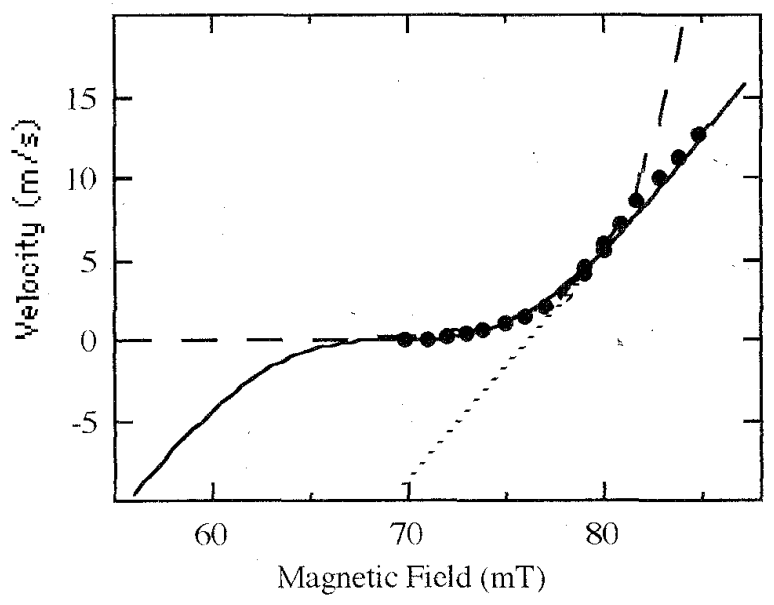

Fig. 3. Domain-wall velocity: (data points) experimental values, (solid line) theoretical prediction, (dashed line) exponential dependence, and (dotted line) linear regime.

For comparison, we have fitted the low-field region to the exponential law Eq. (2). The result is the dashed line in Fig. 3 , where $\mathrm{v}^{\prime}=0.998 \times 10^{-10} \mathrm{~m} / \mathrm{s}$ and $\mu_{0} \mathrm{H}_{O}=3.23 \mathrm{mT}$. We see that it is fairly difficult to distinguish between the exponential and cubic functions in the region between 70 and $80 \mathrm{mT}$, but for $\mu_{\mathrm{o}} \mathrm{H}>80 \mathrm{mT} \mathrm{Eq}$. (9) is superior to Eq. (2). Referring to [5] and taking $\mu_{\mathrm{O}} \mathrm{H}_{\mathrm{O}}=\mathrm{kBT} / 2 \mathrm{VBM}_{\mathrm{S}}, \mu_{\mathrm{O}} \mathrm{M}_{\mathrm{S}}=2$ $\mathrm{T}$ and $\mathrm{VB}=\pi \mathrm{RB}^{2} \mathrm{t}$ yields the reasonable values $4.3 \times 10^{-7}$ $\mu \mathrm{m}^{3}$ and $11.7 \mathrm{~nm}$ for the Barkhausen volume $\mathrm{V}_{\mathrm{B}}$ and the Barkhausen radius $\mathrm{R}_{B}$, respectively. The dotted line in Fig. 3 shows the linear regime $\mathrm{v}=\mu\left(\mathrm{H}-\mathrm{H}_{\mathrm{p}}\right)$.

From Fig. 3 we see that Eq. (9) predicts a plateau where $\mathrm{V} \approx 0$. The width of the plateau, which gives a direct description of the pinning strength of the film, is of order $\mathrm{q} / \mu$. Another feature of Eq. (9) is the prediction of negative domain-wall velocities for negative applied fields. This negative velocity refers to the collapse of domains in a reverse field, which has been observed qualitatively but not analyzed quantitatively. The same behaviour is predicted for zero and small positive fields. In this case the wall-energy term in Eq. (3) dominates the gain in Zeeman energy responsible for domain expansion. Note that the asymmetry of the curve is given by the propagation field $\mathrm{H}_{\mathrm{p}}$, which appears as a shift of the curve's center of gravity.

\section{Conclusions}

In conclusion, we have given a unified description of the mobility of domain walls in thin-films with perpendicular anisotropy. For positive fields, the predictions are in agreement with experimental data, whereas negative fields corresponding to inner hysteresis loops have not yet been investigated quantitatively.

On the other hand, the present approach remains phenomenological not only in the sense of a first-principle theoretical description but also in the sense of a thorough micromagnetic description. Any micromagnetic description starts from quasi-microscopic parameters such as exchange stiffness and local anisotropy, and phenomenological parameters such as the Barkhausen volume must be obtained from calculation rather than being the result of fitting procedures.

\section{APPENDIX}

Consider a domain wall described by the energy

$$
\mathrm{E}_{\mathrm{W}}=\mathrm{E}(\mathrm{x})-\mu_{\mathrm{O}} \mathrm{M}_{\mathrm{S}} \mathrm{HLX}
$$

where $\mathrm{x}$ is the position of the wall. Expanding E into powers of $x$ leads

$$
E=a x+\frac{b}{2} x^{2}+\frac{c}{6} x^{3}+\ldots
$$

A pinning center at $\mathrm{x}=0$ means $\mathrm{a}=0$, since then $\partial \mathrm{E} / \partial \mathrm{x}=0$ at $\mathrm{x}=0$. Putting $\partial \mathrm{E} w / \partial \mathrm{x}=0$ leads a quadratic equation whose solution yields one energy minimum and one energy maximum. The energy difference between these extrema is

$$
\text { or } \begin{aligned}
\Delta \mathrm{E} & =2 \mathrm{~b} / 3\left(\mathrm{~b}^{2} / \mathrm{c}^{2}-2 \mu_{\mathrm{o}} \mathrm{M}_{\mathrm{S}} \mathrm{HL} / \mathrm{c}\right)^{3 / 2} \\
\Delta \mathrm{E} & =\left(2 \mathrm{~b}^{3} / 3 \mathrm{c}^{2}\right)\left(1-\mathrm{H} / \mathrm{H}_{\mathrm{c}}\right)^{3 / 2}
\end{aligned}
$$

Note that this regime corresponds to the fold catastrophe known from bifurcation theory [15].

\section{REFERENCES}

[1] G. Bayreuther, P. Bruno, G. Lugert, and C. Turtur, Phys. Rev. B, vol. 40, p. 7399, 1989.

[2] J. Pommier, P. Meyer, G. Pénissard, J. Ferré, P. Bruno, and D. Renard, Phys. Rev. Lett., vol. 65, p. 2054, 1990

[3] A. Kirilyuk, J. Ferré, and D. Renard, Europhys. Lett., vol. 24, p. $4(3), 1993$.

[4] A. Kirilyuk, J. Ferré, D. Pommier, and D. Renard, J. Magn. Magn. Mater., vol. 121, p. 536, 1993.

[5] J. Ferré, V. Grolier, A. Kirilyuk, J. P. Jamet, and D. Renard, J. Magn. Soc. Jpn., vol. 19-S1, p. 79, 1995.

[6] J. Giergiel and J. Kirschner (in press).

[7] A. Kirilyuk and J. Kirschner, in: Proc. 2nd Int. Symp. Metallic Multilayers, 1996 (in press).

[8] E. Kneller, Ferromagnetismus, Springer, Berlin 1962.

[9] R. Skomski and V. Christoph, phys. stat. sol. (b), vol. 156, p. K149, 1989.

[10] P. Gaunt, J. Appl. Phys., vol. 59, p. 4129, 1986.

[11] D. Givord and M. Rossignol, in: Rare-EarthIron Permanent Magnets, Ed.: J. M. D. Coey, University Press, Oxford 1996, p. 218.

[12] R. Skomski and J. M. D. Coey, Phys. Rev. B, vol. 48 , p. $15812,1993$.

[13] E. C. Stoner and E. P. Wohlfarth, Phil. Trans. Roy. Soc., vol. A240, p. 599, 1948.

[14] R. Skomski, MPI Halle, Seminar at Ringberg Castle, 1996, to be published.

[15] I. Stewart, Rep. Prog. Phys., vol. 45, p. 185, 1982. 